\title{
Assessment Method of the Test Firing Accuracy
}

\author{
Qu Baozhong, Wang $\mathrm{Na}$, Song Jie and Feng Nan
}

92941 troop, China \& post-doctoral scientific research workstation, 92493 troop, China

Keywords: weapon equipment; firing accuracy; Bayes; assessment ;hypothesis inspection

Abstract. The firing error rule of the weapon equipment test was studied, and before and after test's distribution density was confirmed on two conditions with prior information and without prior information. Based on this, the Bayes estimation and hypothesis inspection for the firing accuracy was given, and a method to calculate the probability to fall into a certain area was proposed. These methods can be used for the firing accuracy assessment of ship-to-air missile weapon equipments, and also is valuable for the firing accuracy assessment on the condition of small sample.

\section{Introduction}

In the air firing tests of aviation missile , naval gun weapon and so on, we have to assess the firing accuracy of the weapon equipment test which have the assessment and testing problem involving the root mean square error or falling into the probability. If we have a mount of testing counts, it will be a simple problem to assess in a classical statistics method. However, in fact, because of the economies problem, we always have few amount of test counts, the problem becomes a simple or very simple condition. So we use the Bayes statistical method which is a new method of considering all the test information in every test to calculate and infer a total distribution argument.

\section{Distribution density of firing accuracy before and after the test}

Firing errors law. In the target coordinate system, supposing the system errors is zero and have the same distribution in three directions, same distribution in three directions, that is $\mathrm{x}, \mathrm{y}, \mathrm{z}$ all obey the normal distribution $\mathrm{N}\left(0, \delta_{2}\right)$ and are independent of each other. Practice proves that it is a rational hypothesis, if the system errors is not zero, we can eliminate it by math transformation or in the engineering. That is

$$
r^{2}=x^{2}+y^{2}+z^{2}
$$

As $x^{2}$ distribution theorem, $\frac{r^{2}}{\sigma^{2}}$ obey three-dimensional $\chi^{2}$ distribu- tion, for $\chi_{3}^{2}$, the distribution density is

$$
f_{1}\left(\frac{r^{2}}{\sigma^{2}}\right)=\frac{1}{2^{1.5} \mathrm{~T}\left(\frac{3}{2}\right)}\left(\frac{r^{2}}{\sigma^{2}}\right)^{\frac{3}{2}-1} \mathrm{e}^{\frac{-r^{2}}{\sigma^{2}}}
$$

And $r$ distribution density is

$$
f(r)=f_{1}\left(\frac{r^{2}}{\sigma^{2}}\right) \frac{\mathrm{d}\left(\frac{r^{2}}{\sigma^{2}}\right)}{\mathrm{dr}}
$$

So the firing errors law of aviation missile is

$$
f(r)=\frac{\sqrt{2} r^{2}}{\sqrt{\pi} \sigma^{3}} \mathrm{e}^{-\frac{r^{2}}{2 \sigma^{2}}}, r \geq 0, \sigma \geq 0
$$

The viewpoint of Bayes. To assess the test firing accuracy, analyzing the statistics parameter $\sigma$ is the key. How to assess $\sigma$, the classical method is though collecting the test data then using Matrix method or Maximum likelihood method to assess or test $\sigma$ regarding as a constant quantity. However, the inherence of Bayes is regarding oas a stochastic, before the test, if we have some cognition in it, we call it prior test information and ohas the prior test distribution. Besides, the statistics decision for 
$\sigma$ have relationship with the observations of simple, so through the test in different stages, we have a improvement cognition to $\sigma$.

Distribution density before and after the test in without prior test information. Testing for the first time has no prior test information to use, how to confirm the prior test distribution density, based on the same principle of ignorance, as $\sigma$ is a scale parameter, we order

$$
D=\sigma^{2}
$$

This time prior test distribution is

$$
\Pi(D)=\frac{1}{D}
$$

Through the test for the samples $R=\left(r_{1}, r_{2}, \ldots r_{n}\right)$, independent distribution samples also, among $r_{i}(i=1,2, \ldots n)$ obey $\mathrm{f}(\mathrm{r})$ distribution, given the stochastic $\mathrm{D}$ below, after acquiring the distribution density of $\mathrm{R}$, after test distribution density is

$$
\begin{gathered}
\Pi(D \mid R)=\frac{f(R \mid D) \Pi(D)}{\int_{0}^{1} f(R \mid D) \Pi(D) \mathrm{d} D} \\
\mathrm{f}(\mathrm{R} \mid \mathrm{D}) \Pi(\mathrm{D})=\prod_{\mathrm{i}=1}^{\mathrm{n}}\left(\frac{\sqrt{2}}{\sqrt{\pi}} \frac{r_{i}^{2}}{\sqrt{D^{3}}} \mathrm{e}^{-\frac{r_{i}^{2}}{2 D}}\right) \frac{1}{D}=\left(\frac{\sqrt{2}}{\sqrt{\pi}}\right)^{n}\left(\prod_{i=1}^{n} r_{i}^{2} D^{-\left(\frac{s}{2} n+1\right)} \mathrm{e}^{-\frac{\sum_{i=1}^{n} r_{i}^{2}}{2 D}}\right)
\end{gathered}
$$

Then,

$$
\begin{array}{r}
\left.\mathrm{f}(\mathrm{R} \mid \mathrm{D}) \prod(\mathrm{D})=\prod_{\mathrm{i}=1}^{\mathrm{n}}\left(\frac{\sqrt{2}}{\sqrt{\pi}} \frac{r_{i}^{2}}{\sqrt{D^{3}}} \mathrm{e}^{-\frac{r_{i}^{2}}{2 D}}\right) \frac{1}{D}=\left(\frac{\sqrt{2}}{\sqrt{\pi}}\right)^{n}\left(\prod_{i=1}^{n} r_{i}^{2}\right) D^{-\left(\frac{s}{2} n+1\right)} \mathrm{e}^{-\frac{\sum_{i=1}^{n} r_{i}^{2}}{2 D}}\right) \\
\left.\Pi(\mathrm{D} \mid \mathrm{R})=\frac{\left(\frac{1}{2} \sum_{i=1}^{n} r_{i}^{2}\right)^{\frac{s}{2} n}}{\Gamma(s n / 2)} D^{-\left(\frac{s}{2} n+1\right)} \mathrm{e}^{-\frac{\sum_{i=1}^{n} r_{i}^{2}}{2 D}}\right)
\end{array}
$$

Take notice of expression of the opposite Gamma function

$$
g(x ; \alpha, \beta)=\frac{\alpha^{\beta}}{\Gamma(\beta)} x^{-(\beta+1)} \mathrm{e}^{-\frac{\alpha}{x}}, x>0 ; \alpha, \beta>0
$$

So the distribution density of without prior test information is

$$
\Pi(D \mid R)=g\left(D ; \frac{1}{2} \sum_{i=1}^{n} r_{i}^{2}, \frac{3}{2} n\right)
$$

Distribution density before and after the test in prior test information. We have discussed the situation of without prior test before, while in fact having the prior test information is in the majority. As a lot of data acquired through emulation can also by the information in the last test. So the distribution in prior test can be confirmed by using the maximum entropy method or self-help methods. We can also use the after test distribution in the last time as the before test distribution in the next time. So we suppose before test distribution is

$$
\Pi(D)=g\left(D ; \alpha_{0}, \beta_{0}\right)
$$

Through the test we see $R=\left(r_{1}, r_{2}, \ldots r_{n}\right)$, independent distribution samples also, among $r_{i}(i=1,2, \ldots n)$ obey $\mathrm{f}(\mathrm{r})$ distribution, this is after test distribution density below

$$
\left.\Pi(\mathrm{D} \mid \mathrm{R})=\frac{\left(\alpha_{o}+\frac{1}{2} \sum_{i=1}^{n} r_{i}^{2}\right)^{\beta_{0}+\frac{3}{2} n}}{\Gamma\left(\beta_{o}+\frac{3}{2} n\right)} D^{-\left(\beta_{o}+\frac{3}{2} n+1\right)} \mathrm{e}^{-\frac{1}{D}\left(\alpha_{o}+\frac{1}{2} \sum_{i=1}^{n} r_{i}^{2}\right)}\right)
$$

Let 


$$
\alpha_{1}=\alpha_{0}+\frac{1}{2} \sum_{i=1}^{n} r_{i}^{2}, \quad \beta_{1}=\beta_{0}+\frac{3}{2} n
$$

The after test distribution density is

$$
\Pi(D)=g\left(D ; \alpha_{1}, \beta_{1}\right)
$$

We can see from the discussion, the firing accuracy $\mathrm{D}$ of aviation missile before and after test have a conjugated distribution, in other words $\Pi(D)$ have a same distribution form.

\section{Bayesian assession of fire precision.}

Classical method to fire precision to estimate is applied for a long time. To the instance that the arm equipment test swatch is less, how to consider prior test information, underside there are Bayesian assession of ship-to-air missile fire precision and hypothetical inspection method.

Bayesian evaluation of fire precision. Under square damnify,

$$
\hat{D}_{B}=\mathrm{E}[D \mid R]=\int_{0}^{\infty} D \prod(D \mid R) \mathrm{d} D=\int_{0}^{\infty} D \frac{\alpha_{1}^{\beta_{1}-1}}{\Gamma\left(\beta_{1}\right)} D^{-\left(\beta_{1}-1\right)} \mathrm{e}^{-\frac{\alpha_{1}}{D}} \mathrm{~d} D=\frac{\alpha_{1}}{\beta_{1}-1}
$$

Thereinto $\Gamma\left(\beta_{1}\right)=\left(\beta_{1}-1\right) \Gamma\left(\beta_{1}-1\right), \quad \int_{0}^{\infty} \mathrm{g}\left[D ; \alpha_{1}, \beta_{1}-1\right] \mathrm{d} D=1$

Difference of Classical and Bayesian assession is great. Underside adopting Classical maximum likelihood method to estimate collectivity parameter, difference of two methods is clear.

$$
\mathrm{L}(\mathrm{D})=\prod_{\mathrm{i}=1}^{\mathrm{n}} \mathrm{f}\left(\mathrm{r}_{\mathrm{i}} \mid \mathrm{D}\right)=\left(\frac{\sqrt{2}}{\sqrt{\pi}}\right)^{n} \prod_{i=1}^{n} r_{i}^{2} D^{-\left(\frac{3}{2} n\right)} \mathrm{e}^{-\frac{\sum_{i=1}^{n} r_{i}^{2}}{2 D}}
$$

Let

$$
\frac{\partial \mathrm{L}(D)}{\partial D}=-\frac{3}{2} n \frac{1}{D}+\frac{1}{2}\left(\sum_{i=1}^{n} r_{i}^{2}\right) \frac{1}{D^{2}}=0
$$

Maximum likelihood method of fire precision $\mathrm{D}$ is

$$
\hat{D}_{L}=0.5 \sum_{i=1}^{n} r_{i}^{2} / \frac{3}{2} n
$$

If test swatch $n$ is enough great, $n \circledast 1$, bayesian and classical assession have following connection.

$$
\begin{aligned}
& \hat{D}_{B}=\frac{\alpha_{1}}{\beta_{1}-1}=\frac{\alpha_{0}+\frac{1}{2} \sum_{i=1}^{n} r_{i}^{2}}{\beta_{0}+\frac{3}{2} n-1} \rightarrow \frac{\frac{1}{2} \sum_{i=1}^{n} r_{i}^{2}}{\frac{3}{2} n}=\hat{D}_{L} \\
& \text { viz. } \mathrm{D}_{\mathrm{z}} \rightarrow \mathrm{D}_{\mathrm{L}} \text {, แ } \text { 准 } 1
\end{aligned}
$$

By this token, Classical statistical maximum likelihood method estimation becomes especial instance of bayesian assession.At the same time, account for that when test swatch $n$ is enough great, information in swatch is sufficient,not considering prior test information.

Calculation of fire droping into probability. Calculating fire droping into probability needs considering semidiameter of droping into sphere. Following present droping into probability calculation method of semidiameter $\sigma$ of sphere.

$$
P_{m \sigma}=\frac{\sqrt{2}}{\sqrt{\pi}} \int_{0}^{m \sigma} \frac{r^{2}}{\sigma^{s}} \mathrm{e}^{-\frac{r^{2}}{2 \sigma^{2}}} \mathrm{~d} r=\frac{\sqrt{2} m}{\sqrt{\pi}} \mathrm{e}^{-\frac{m^{2}}{2}}+2[\phi(m)-\phi(0)]
$$

Coordinating to receive

$$
P_{m \sigma} \triangleq 2 \phi(m)-0.798 m \mathrm{e}^{-\frac{m^{2}}{2}}-1, m>0
$$

When $\mathrm{m}=2,3, P_{2 \sigma} \triangleq 0.74$ and $P_{3 \sigma} \triangleq 0.97$.

Bayesian hypothetical inspection of fire precision. Known $r$ obey $\mathrm{f}(\mathrm{r} \mid \mathrm{D})$, present statistical hypothesis is

$$
\left\{\begin{array}{l}
\mathrm{H}_{0}: D \leq D_{0} \\
\mathrm{H}_{1}: D>D_{0}
\end{array}\right.
$$


Thereinto $D=\sigma^{2}$, and $D \leq D_{0}$ is $H_{0}, \quad D>D_{0}$ is $H_{1}$ 。 Defining permanent damnify function $L\left(D, a_{j}\right)=C_{i j}$, when $D \in H_{i}, \quad \mathrm{i}, \mathrm{j}=0,1$, thereinto $C_{k k}>C_{i j}, \mathrm{i} \neq \mathrm{j}, \mathrm{k}, \mathrm{i}, \mathrm{j}=0,1$ 。 $a_{0}$ expresses decision-making action of adopting $H_{0}$ hypothesis, $a_{1}$ expresses decision-making action of adopting $H_{1}$ hypothesis. All of them are functions of swatch.

Now, if obtain $R=\left(r_{1}, r_{2}, \ldots r_{n}\right)$ swatch, $a_{0}$ prior test expectation damnify of decision-making is

$$
\mathrm{E}\left[\mathrm{L}\left(\mathrm{D}, \partial_{0}\right) \mid \mathrm{R}\right]=\int_{\mathrm{H}} \mathrm{L}\left(\mathrm{D}, \partial_{\mathrm{o}}\right) \Pi(\mathrm{D} \mid \mathrm{R}) \mathrm{d}(\mathrm{D})=\mathrm{C}_{00} \mathrm{P}\left(\mathrm{H}_{0} \mid \mathrm{R}\right)+\mathrm{C}_{01} \mathrm{P}\left(\mathrm{H}_{1} \mid \mathrm{R}\right)
$$

In common

$$
\mathrm{E}\left[\mathrm{L}\left(\mathrm{D}, \partial_{1}\right) \mid \mathrm{R}\right]=\mathrm{C}_{10} \mathrm{P}\left(\mathrm{H}_{0} \mid \mathrm{R}\right)+\mathrm{C}_{11} \mathrm{P}\left(\mathrm{H}_{1} \mid \mathrm{R}\right)
$$

When $\mathrm{E}\left[\mathrm{L}\left(\mathrm{D}, \partial_{0}\right) \mid \mathrm{R}\right]<\mathrm{E}\left[\mathrm{L}\left(\mathrm{D}, \partial_{1}\right) \mid \mathrm{R}\right]$, adopt $H_{0}$ hypothesis, contrariwise adopt $H_{1}$ hypothesis, decision-making inequation is

$$
\frac{\mathrm{P}\left(\mathcal{H}_{1} \mid \mathrm{R}\right)}{\mathrm{P}\left(\mathcal{H}_{0} \mid \mathrm{R}\right)} \underset{\text { acci: }}{\operatorname{ar} \mathrm{H}_{1}} \frac{\mathrm{C}_{10}-\mathrm{C}_{00}}{\mathrm{C}_{01}-\mathrm{C}_{11}}
$$

Thereinto acc.is the abbreviation of accept, denoting adopting D Due to

$$
\mathrm{P}\left(\mathcal{F}_{\odot} \mid \mathrm{R}\right)=1-\mathrm{P}\left(\mathcal{F}_{1} \mid \mathrm{R}\right)
$$

Coordinate

$$
\mathrm{P}\left(\mathcal{K}_{1} \mid \mathrm{R}\right) \underset{\text { acc: } \mathrm{H}_{1}}{\mathrm{C}_{\mathrm{O} 1}+\mathrm{C}_{10}-\mathrm{C}_{00}-\mathrm{C}_{11}}
$$

Critical area of corresponding bayesian assession, namely area of refusing $H_{0}$ hypothesis is

$$
\mathrm{D}=\left\{\mathrm{R}=\left(\mathrm{r}_{1}, \mathrm{r}_{2}, \mathrm{r}_{3}, \ldots, \mathrm{r}_{\mathrm{n}}\right) ; \mathrm{P}\left(\mathcal{H}_{1} \mid \mathrm{R}\right) \underset{\mathrm{acc}: \mathrm{H}_{1}}{>} \frac{\mathrm{C}_{\mathrm{O} 1}-\mathrm{C}_{11}}{\mathrm{C}_{\mathrm{O}}+\mathrm{C}_{10}-\mathrm{C}_{00}-\mathrm{C}_{11}}\right\}
$$

Due to

$$
\mathrm{P}\left(\mathrm{H}_{1} \mid \mathrm{R}\right)=\int_{\mathrm{D}_{0}}^{\infty} \Pi(\mathrm{D} \mid \mathrm{R}) \mathrm{dD}=\int_{\mathrm{D}_{0}}^{\infty} \frac{\alpha_{1}^{\beta_{1}}}{\Gamma\left(\beta_{1}\right)} D^{-\left(\beta_{1}+1\right)} \mathrm{e}^{-\frac{\alpha_{1}}{D}} \mathrm{~d} D=\mathrm{K}_{2 \beta_{1}}\left(\frac{2 \alpha_{1}}{D_{0}}\right)
$$

Due to

Thereinto, obtaining $\mathrm{K}_{2 \beta_{1}}\left(\frac{2 \alpha_{1}}{D_{0}}\right)$ is by checking table of $2 \beta_{1}$ dimension $\chi^{2}(\cdot)$, is

$$
\begin{gathered}
\frac{2 \alpha_{1}}{D_{0}} ; K_{2 K_{2}}^{-1}\left(\frac{C_{01}-C_{11}}{C_{01}+C_{10}-C_{00}-C_{11}}\right) \\
\sum_{i=1}^{n} r_{i}^{2} \geqslant D_{0} K_{2 K_{1}}^{-1}\left(\frac{C_{01}-C_{11}}{C_{01}+C_{10}-C_{00}-C_{11}}\right)-2 \alpha_{0}
\end{gathered}
$$

Critical area of corresponding bayesian assession, namely area of refusing $H_{0}$ hypothesis is

$$
\mathrm{D}=\left\{\mathrm{R}=\left(\mathrm{r}_{1}, \mathrm{r}_{2}, \mathrm{r}_{2}, \ldots \ldots, \mathrm{r}_{\mathrm{n}}\right): \sum_{\mathrm{i}=1}^{\mathrm{n}} \mathrm{r}_{1}^{2}>\mathrm{D}_{0} \mathrm{~K}_{2 \mathrm{~S}_{1}}^{-1}\left(\frac{\mathrm{C}_{01}-\mathrm{C}_{11}}{\mathrm{C}_{01}+\mathrm{C}_{10}-\mathrm{C}_{00}-\mathrm{C}_{11}}\right)-2 \alpha_{0}\right\}
$$

\section{Conclusions}

Guideline of fire precision is among important tactics and technology guideline of the arm equipment, and is also the base of assessing guideline for kill rate. Through achieving test information on different phases and format, on the base of accomplishing correlative and absolute same distribution identifition, adopt the method of Bayesian test statistics, and accomplish evaluating and tentativly proving guideline of fire precision. This method that is primely applied to test and identifition of 
ship-to-air missile and ship-artillery, and is also popularized to apply test and identifition of other arm equipment, can lessen test swatch, and improve test believe level.

\section{References}

[1] Jinkui Zhang and Xuemei Tang: BAYES method. (Publishing company of nscience and technologyational defence university, Chang sha1993). In Chinese

[2] Baozhong Qu and Xiaofeng Sun: Navy tactics missile test and identifition. (national defence industry publishing company, Beijing 2005). In Chinese

[3] Mingyao Xiao: Experimentation error evaluation and data disposal. (science publishing company, Beijing 1984). In Chinese

[4] Zhong shan da xue: Probability theoretics and symbolic Stat. (dumb millions education publishing company, Beijing 1980). In Chinese 